\title{
Nasal Glioma: Prenatal Diagnosis and Multidisciplinary Surgical Approach
}

\author{
Olubunmi Ajose-Popoola, B.A., ${ }^{1}$ Harrison W. Lin, M.D., ${ }^{2}$ \\ V. Michelle Silvera, M.D.,' ${ }^{3}$ Lisa A. Teot, M.D., ${ }^{4}$ Joseph R. Madsen, M.D., 5 \\ John G. Meara, M.D., D.M.D., M.B.A., ${ }^{6}$ and Reza Rahbar, M.D., D.M.D.7
}

Nasal gliomas are congenital, nonmalignant rests of neuroglial tissue that typically present as a craniofacial mass. The differential diagnosis of such masses includes lesions that often require the involvement of various surgical subspecialties, including otolaryngology, neurosurgery, plastic surgery, and ophthalmology. Early surgical excision of these masses is advised to minimize nasal and craniofacial distortion. Accordingly, early diagnosis and management planning are paramount, and advances in prenatal imaging are creating a new role for obstetricians and radiologists in the initiation of diagnostic and therapeutic interventions. We describe the case history of a young patient found to have a craniofacial mass on routine prenatal ultrasound and subsequently managed with a multidisciplinary team approach.

KEYWORDS: Nasal glioma, craniofacial mass, prenatal imaging

$\mathrm{N}$ asal gliomas are rare and benign congenital lesions of the craniofacial region composed of heterotopic masses of neuroglial tissue. These masses are believed to be the consequence of incomplete closure of the anterior fontanelle between the nasal and frontal bones, resulting in an abnormal connection between embryonic ectodermal and neuroectodermal elements. Although benign in nature, gliomas and other congenital midline craniofacial malformations are often cosmetically and/or clinically unfavorable, and accordingly, total excision is the treatment of choice. Early surgical intervention has been encouraged to minimize nasal and craniofacial distortion and early diagnosis and assessment is therefore paramount. Advances in pre- and postnatal imaging have introduced new roles for specialties not traditionally intimately involved in nasal glioma management, including obstetrics and radiology. Here we review a case of a young patient with a nasal glioma diagnosed prenatally by the obstetrician and radiologist and subsequently addressed postnatally by the more traditional specialties involved in midline craniofacial mass management, including otolaryngology, neurosurgery, and plastic surgery.

\section{CASE REPORT}

A 3-day-old boy presented to our institution for an evaluation of a nasal mass. He had been born to a healthy 31-year-old woman who had undergone a routine prenatal ultrasound (Fig. 1), which revealed the mass. Prenatal magnetic resonance imaging (MRI) demonstrated an anterior craniofacial mass isointense to brain parenchyma separated from the frontal lobes by a distinct cerebrospinal fluid plane (Fig. 2). On
${ }^{1}$ Harvard Medical School; ${ }^{2}$ Department of Otolaryngology, Massachusetts Eye and Ear Infirmary; Departments of ${ }^{3}$ Radiology; ${ }^{4}$ Pathology; ${ }^{5}$ Neurosurgery; ${ }^{6}$ Plastic and Oral Surgery; and ${ }^{7}$ Otolaryngology and Communication Enhancement, Children's Hospital Boston, Boston, Massachusetts.

Address for correspondence and reprint requests: Harrison W. Lin, M.D., Massachusetts Eye and Ear Infirmary, 243 Charles Street, Boston, MA 02114 (e-mail: harrison_lin@meei.harvard.edu).
Skull Base Rep 2011;1:83-88. Copyright (C) 2011 by Thieme Medical Publishers, Inc., 333 Seventh Avenue, New York, NY 10001, USA. Tel: +1(212) 584-4662.

Received: January 5, 2011. Accepted after revision: April 22, 2011. Published online: August 3, 2011.

DOI: http://dx.doi.org/10.1055/s-0031-1284210.

ISSN 2157-6971. 

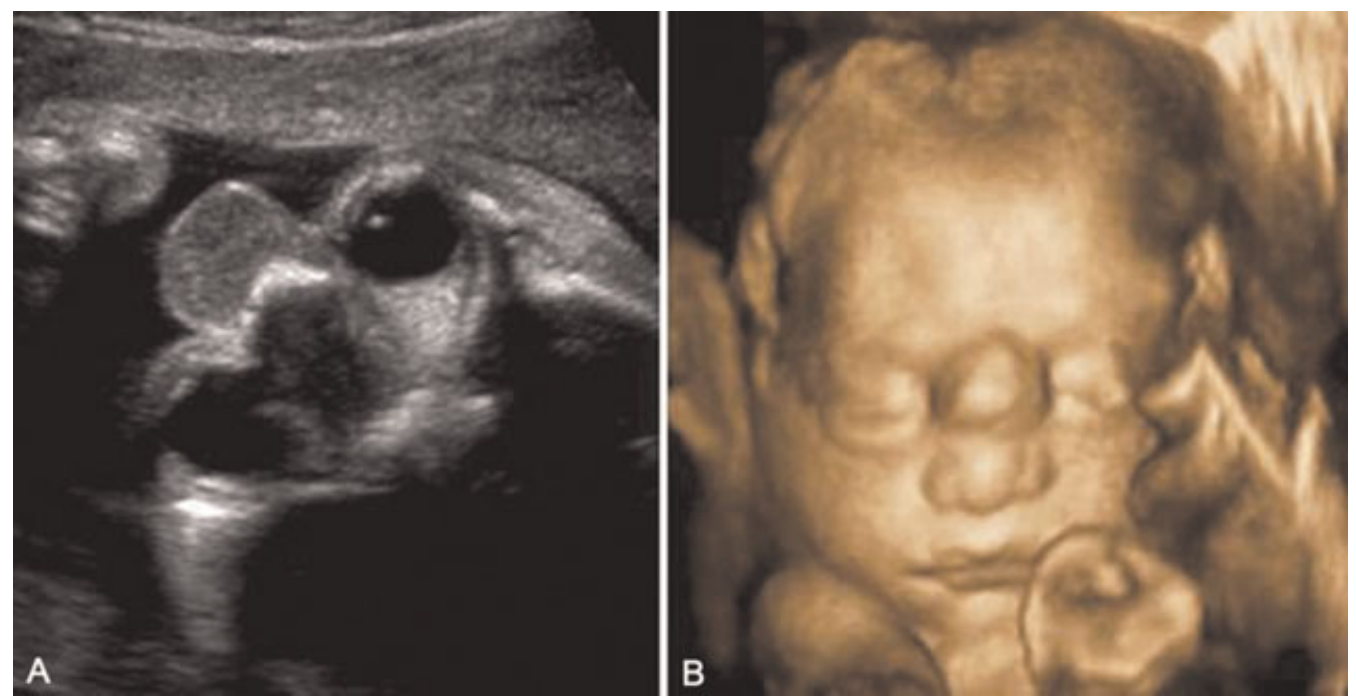

Figure 1 (A) Prenatal ultrasound and (B) three-dimensional reconstruction demonstrating the nasal mass.

examination, he was found to be well appearing and had no abnormalities of heart, lungs, abdomen, palate, or spine. However, a bluish $2.5 \times 2.5 \mathrm{~cm}$ soft tissue skincovered mass in the nasal-glabellar region was noted (Fig. 3A, B). The mass was nonpulsatile, without bruit, and incompressible.

Postnatal MRI revealed a $2.8 \times 1.8 \times 1.8 \mathrm{~cm}$ mass centered in the midline overlying the bridge of the nose with a potential intracranial connection between the mass and anterior cranial fossa through a bony defect (Fig. 4). The mass demonstrated no enhancement,

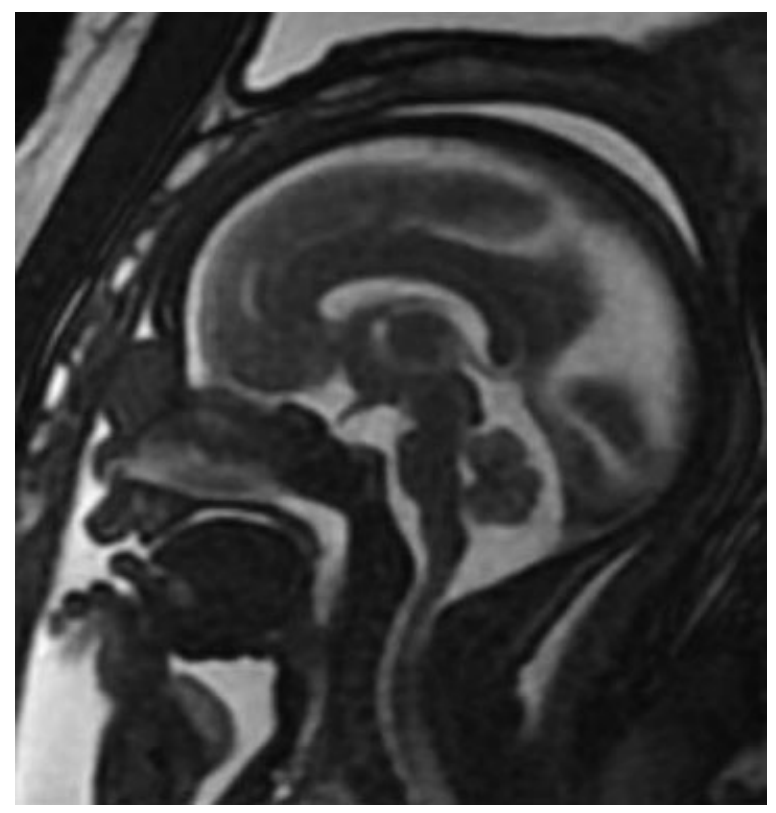

Figure 2 Subsequent fetal T2-weighted sagittal MRI revealing an anterior craniofacial mass separated from the brain by a uniform cerebrospinal fluid plane. followed brain parenchymal signal intensity on both T1- and T2-weighted sequences and had a lobulated/ gyral marginal contour. A computed tomography $(\mathrm{CT})$ scan at 77 days of life demonstrated a $2.8 \times 2.0 \times 2.0 \mathrm{~cm}$ mass isodense to the brain parenchyma with a connection to the intracranial vault through an $8 \times 6 \mathrm{~mm}$ bony defect at the glabella and the metopic suture (Fig. 5). The patient was subsequently taken to the operating room where the mass was excised through a midline nasal incision (see Fig. 3C, D). No patent connection to the intracranial compartment was noted. Histopathology revealed moderately cellular, well-differentiated neuroglial tissue without evidence of microvascular proliferation, necrosis, leptomeninges, ependyma, or choroid plexus. Large neurons, including binucleate and multinucleate forms, were noted (Fig. 6).

\section{DISCUSSION}

Nasal gliomas are nonmalignant rests of heterotopic neural tissue and are one of several types of congenital midline nasal masses, a group of anomalies with an estimated incidence of 1:20,000 to 40,000 births. ${ }^{1}$ While encephaloceles are the most common congenital midline nasal mass, dermoids, epidermoids, gliomas, teratomas, hemangiomas are less commonly seen. Nasal gliomas are typically located in the region of the glabella, but can extend down to the nasal tip. They may manifest extranasally $(60 \%)$, intranasally $(30 \%)$, or both $(10 \%)$, and have been found in the nasopharynx, paranasal sinuses, tonsillar fossa, scalp, and orbit. ${ }^{2}$ Approximately 250 cases have been reported in the literature. ${ }^{3}$

While the etiology of this lesion is unknown, multiple theories regarding its origin abound. The most widely accepted hypothesis is the "encephalocele theory," which states that both the encephalocele and 

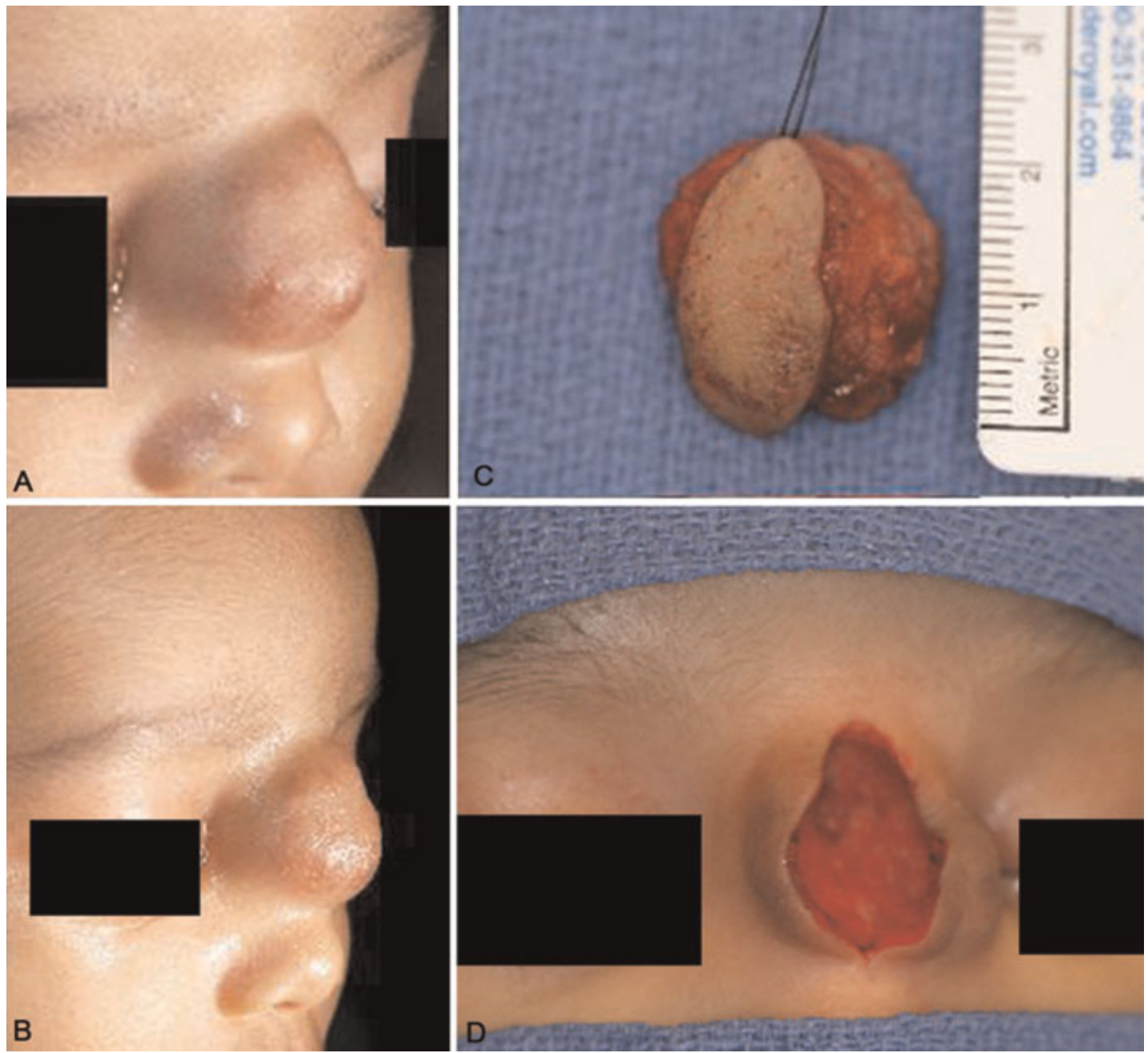

Figure $3(A, B)$ Preoperative views of the mass. (C, D) The mass was excised en bloc with a fusiform cuff of skin to optimize the cosmetic appearance of the wound closure.
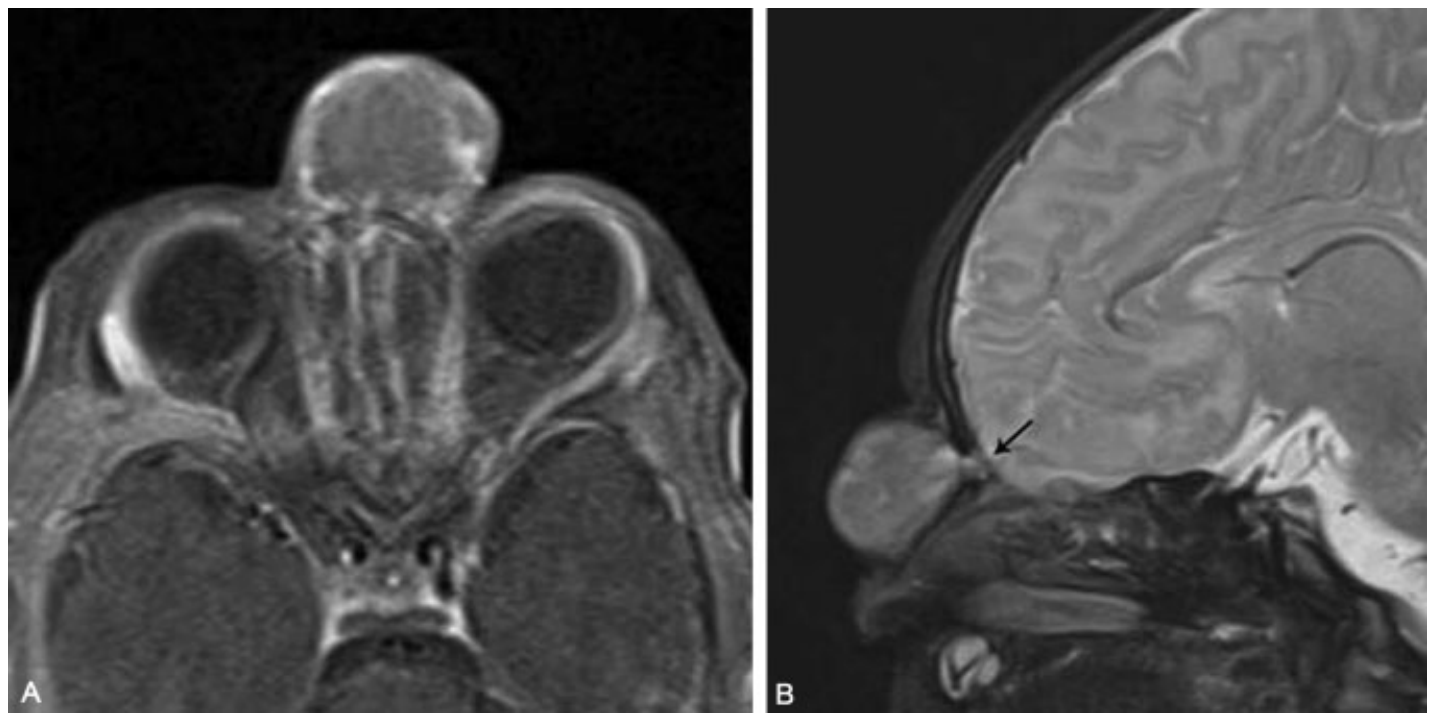

Figure 4 Postnatal T1-weighted axial (A) and T2-weighted sagittal (B) MRI revealing a nonenhancing midline mass with a potential intracranial connection with the anterior cranial fossa (arrow) 


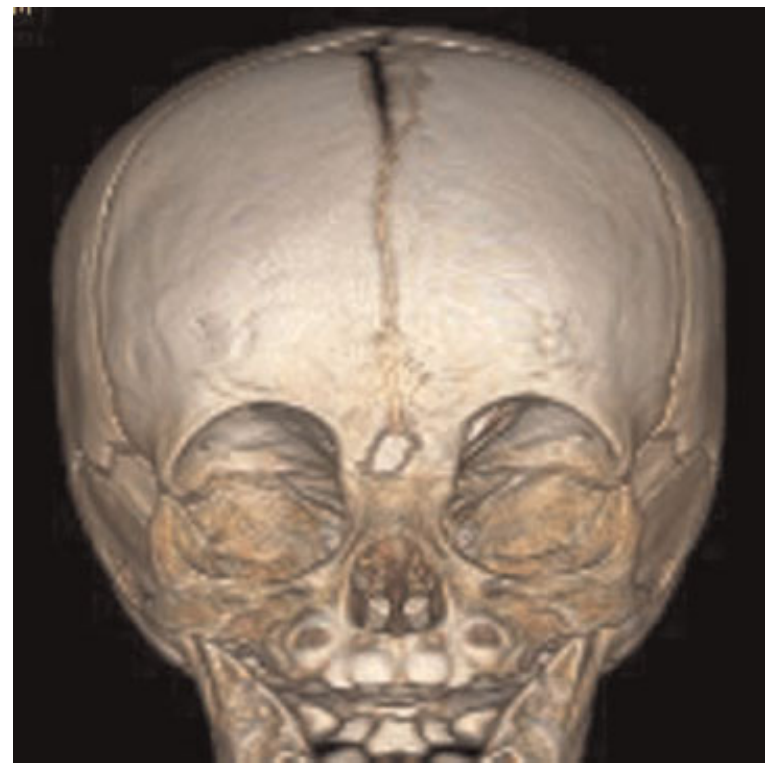

Figure 5 CT three-dimensional reconstruction demonstrating an $8 \times 6 \mathrm{~mm}$ bony defect at the glabella and the metopic suture.

glioma develop secondary to a failure of regression of the forebrain dural protrusion through the foramen cecum or fonticulus frontalis. ${ }^{2}$ This failure of regression is postulated to result from a sustained connection between neural and surface ectoderm, due to insufficient apoptosis at the final closure site of the rostral neuropore. ${ }^{4}$ In encephaloceles, the connection to the brain is maintained, while in gliomas this connection is obliterated, leaving a nest of tissue. In 15 to $20 \%$ of glioma cases, a fibrous stalk connection between the heterotopic tissue and the intracranial compartment occurs. ${ }^{1,5}$ Although these masses have been noted to occur in patients with cleft palates, a review of the literature by Uemura and

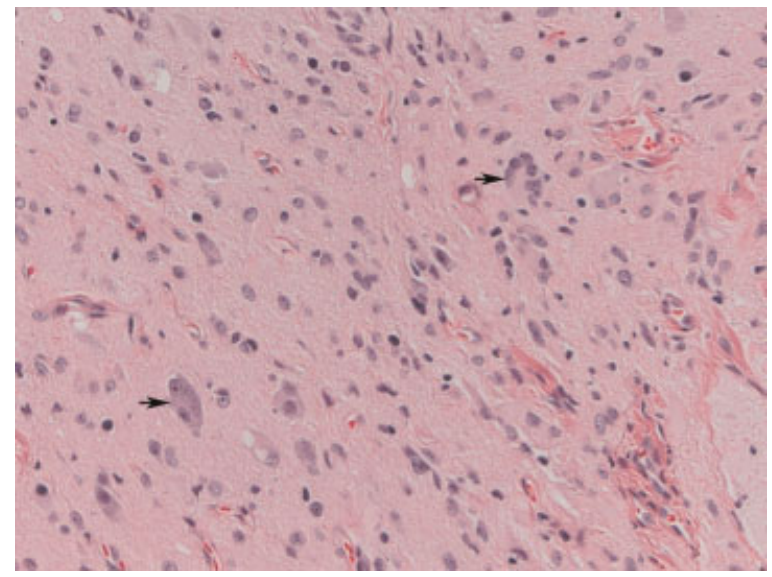

Figure 6 Histopathology demonstrating moderately cellular, well-differentiated neuroglial tissue. Large neurons, including binucleate and multinucleate forms, are shown (arrows). colleagues failed to demonstrate other associated malformations or risk factors. ${ }^{6}$

Given modern advances in the quality and resolution of prenatal ultrasonography and MRI, the diagnosis and therapeutic planning of nasal gliomas, encephaloceles, and other craniofacial masses have recently transitioned from the postnatal to the prenatal period. Routine ultrasound and three-dimensional reconstructions of the images have provided obstetricians and radiologists the capacity to detect these lesions and to obtain additional diagnostic imaging. Typically, a fetal MRI can subsequently be performed to provide superior soft tissue definition to assess the olfactory bulbs, frontal lobes, cribriform plate, crista galli, and other intracranial abnormalities or connections with the mass. ${ }^{7}$ Neurosurgical consultation should be obtained with the presence of any suspicion for such anomalies or a patent communication between the mass and the intracranial vault. MRI may also help narrow the differential diagnosis of a midline craniofacial malformation seen on ultrasound, which includes glioma, encephalocele, teratoma, dermoid cyst, dacryocystocele, retinoblastoma, and hemangioma. A more limited differential diagnosis can facilitate pre- and postnatal consultations with relevant surgical specialties, including ophthalmologists, neurological surgeons, and otolaryngologists, and thereby better address parental questions and anxiety. Nasal gliomas will often appear isointense to gray matter on T1-weighted imaging, heterogeneous on T2-weighted imaging, and nonenhancing to slightly enhancing with contrast. ${ }^{8}$ Moreover, MRI can evaluate the spinal column for a meningoencephalocele or other midline abnormalities and assess the aerodigestive tract for potential obstructions. Given that neonates are obligate nasal breathers and that postpartum airway obstruction has been reported in newborns with nasal and nasopharyngeal neuroglial heterotopias, a careful radiologic assessment of the airway on the prenatal MRI is paramount. ${ }^{9,10}$ If obstruction is anticipated, definitive airway interventions, such as immediate postpartum intubation or ex utero intrapartum treatment procedures can be discussed and planned with the obstetrician.

The postnatal clinical presentation of patients with nasal gliomas will be dependent on the location of the lesion. As in this case, extranasal gliomas present as incompressible masses, most often in the glabellar region. They often have bluish hue and a telangiectatic surface, which may precipitate misdiagnosis as a hemangioma or vascular malformation. ${ }^{11}$ Intranasal and mixed gliomas typically arise from the nasal septum or lateral nasal wall near the middle turbinate and therefore may present with symptoms of nasal obstruction. ${ }^{12}$ An important distinction between nasal gliomas and encephaloceles found on physical examination is the absence of size variation of nasal gliomas with an increase in 
intracranial pressure. Nasal gliomas should not change in size with compression of the internal jugular vein due to the absence of a patent connection with the central nervous system. In contrast, encephaloceles can vary in size with changes in intracranial pressure (Furstenberg sign).

High resolution multiplanar MRI is the preferred modality for evaluating these lesions postnatally as it allows for optimal visualization of any intracranial extension. ${ }^{11}$ However, it is limited in its ability to distinguish a true patent communication to the intracranial fossa from a fibrous stalk connection that is occasionally seen with nasal gliomas. CT is also helpful for assessing bony defects, although this modality may be of variable utility in young infants as the bony architecture is not fully formed and the evolving ossification of the frontal, nasal, and ethmoid bones can lead to a false interpretation of bony dehiscence. ${ }^{11}$

As these lesions lack malignant potential, nasal gliomas are treated with surgery alone. Extranasal gliomas can be excised via lateral rhinotomy, external rhinoplasty, midline nasal incision, or a bicoronal incision. ${ }^{1}$ Minimally invasive endoscopic surgery is emerging as the mainstay for treatment of intranasal gliomas, as angled endoscopy allows for improved visualization of the cribriform plate. This modality is anticipated to replace craniotomy as the procedure of choice for treating an intranasal or mixed glioma with an intracranial extension. ${ }^{13}$ For both intranasal and extranasal gliomas, removal of the stalk is imperative not only to decrease the rate of recurrence but also to minimize the chances of a cerebrospinal fluid leak and subsequent meningitis. Operative involvement of neurosurgical and plastic surgical services may be indicated based on the size, location, and extent of the lesion to optimize the extirpation of the mass and cosmetic outcome. Recurrence rates of 4 to $10 \%$ have been reported with this anomaly. ${ }^{5}$ Surgical excision of these lesions should be initiated as early as possible to minimize the chances of complications, such as distortion of the nasal architecture.

Histopathological diagnosis is made by observation of mature glial cells, interwoven with fibrovascular connective tissue. Occasionally focal areas of calcification are also seen. ${ }^{5}$ The presence of leptomeninges, ependyma, or choroid plexus would support a diagnosis of encephalocele rather than a diagnosis of nasal glioma.

Although the histopathology of nasal gliomas has been well described, and the etiology theorized, studies evaluating for and identifying risk factors for the development of these lesions are lacking. This is likely due in large part to the rarity of these cases. Current research into risk factors for development of encephaloceles, as well as other neural tube defects, has explored the role of environmental and genetic factors. Notably, the inci- dence of anterior encephaloceles in the children of Thailand and India has been reported to be as high as 8 times that of the Western hemisphere, ${ }^{4}$ and accordingly clinical, embryologic, and epidemiologic research has focused on identifying possible early teratogenic insults. Moreover, Burren and colleagues found that mice with a mutation in the $P A X 3$ gene, which codes for a transcription factor involved in neural tube closure, had an increase in susceptibility to neural tube defects when folate was restricted. ${ }^{14}$ Folate-restricted mice without the mutation were found to have fewer neural tube defects. These findings allude to the importance of environmental and genetic interactions in the pathogenesis of these defects. However, whether these findings can be extrapolated to nasal gliomas remains to be seen.

\section{CONCLUSION}

Nasal gliomas and other anterior craniofacial masses are uncommon lesions. Nevertheless, when present, these masses are uniformly managed surgically, with early operative intervention believed to correlate with more favorable aesthetic outcomes. Advances in imaging technologies have provided clinicians from various specialties with an opportunity to facilitate earlier diagnoses and timely surgical consultations. Pre- and postnatal discussion and counseling with expecting families and new parents by a multidisciplinary team may minimize family anxiety and optimize patient outcomes.

\section{REFERENCES}

1. Rahbar R, Resto VA, Robson CD, et al. Nasal glioma and encephalocele: diagnosis and management. Laryngoscope 2003;113(12):2069-2077

2. Riffaud L, Ndikumana R, Azzis O, Cadre B. Glial heterotopia of the face. J Pediatr Surg 2008;43(12):e1-e3

3. Niedzielska G, Niedzielski A, Kotowski M. Nasal ganglioglioma-difficulties in radiological imaging. Int J Pediatr Otorhinolaryngol 2008;72(2):285-287

4. Hoving EW. Nasal encephaloceles. Childs Nerv Syst 2000; 16(10-11):702-706

5. Patterson K, Kapur S, Chandra RS. "Nasal gliomas" and related brain heterotopias: a pathologist's perspective. Pediatr Pathol 1986;5(3-4):353-362

6. Uemura T, Yoshikawa A, Onizuka T, Hayashi T. Heterotopic nasopharyngeal brain tissue associated with cleft palate. Cleft Palate Craniofac J 1999;36(3):248-251

7. Grzegorczyk V, Brasseur-Daudruy M, Labadie G, Cellier C, Verspyck E. Prenatal diagnosis of a nasal glioma. Pediatr Radiol 2010;40(10):1706-1709

8. De Biasio P, Scarso E, Prefumo F, Odella C, Rossi mid trimester. Ultrasound Obstet Gynecol 2006;27(5): 571-573

9. Pakkasjärvi N, Salminen P, Kalajoki-Helmiö T, Rintala R, Pitkäranta A. Respiratory distress secondary to nasopharyngeal 
glial heterotopia. Eur J Pediatr Surg 2008;18(2):117118

10. Husein OF, Collins M, Kang DR. Neuroglial heterotopia causing neonatal airway obstruction: presentation, management, and literature review. Eur J Pediatr 2008;167(12): 1351-1355

11. Hedlund G. Congenital frontonasal masses: developmental anatomy, malformations, and MR imaging. Pediatr Radiol 2006;36(7):647-662; quiz 726-727
12. Morgan DW, Evans JN. Developmental nasal anomalies. J Laryngol Otol 1990;104(5):394-403

13. Wu CL, Tsao LY, Yang AD, Chen MK. Endoscopic surgery for nasal glioma mimicking encephalocele in infancy. Skull Base 2008;18(6):401-404

14. Burren KA, Savery D, Massa V, et al. Gene-environment interactions in the causation of neural tube defects: folate deficiency increases susceptibility conferred by loss of PAX3 function. Hum Mol Genet 2008;17(23):3675-3685 